\title{
Tripterygium glycosides induce premature ovarian failure in rats by promoting p53 phosphorylation and activating the serine/threonine kinase 11-p53-p21 signaling pathway
}

\author{
TE LIU $^{1,2^{*}}$, LINA ZHANG $^{1 *}$, SUWEI WANG ${ }^{1}$, CHUAN CHEN $^{2}$ and JIN ZHENG ${ }^{1}$ \\ ${ }^{1}$ Department of Gynecology; ${ }^{2}$ Shanghai Geriatric Institute of Chinese Medicine, Longhua Hospital, \\ Shanghai University of Traditional Chinese Medicine, Shanghai 200031, P.R. China
}

Received December 13, 2014; Accepted January 20, 2015

DOI: $10.3892 / \mathrm{etm} .2015 .2498$

\begin{abstract}
Premature ovarian failure (POF) is a typical pathological disease of the reproductive system in aging females. Infection, inflammation, immune abnormalities, genetic mutation, radiotherapy and chemotherapy can cause POF. Tripterygium glycosides (TGs) are a component extracted from the Chinese herb Tripterygium wilfordii Hook. f., also known as Huangteng. Although TGs have been used to treat various diseases, drug resistance and toxicity can affect patients. The aim of the present study was to investigate the mechanism of TG-induced POF in rats. The rats were treated with different concentrations of TG, and pathology assays showed that the TG-induced POF was predominantly composed of interstitial cells in a fibrous matrix with a reduced number of follicles at each stage and an increased number of collapsed oocytes. Furthermore, reverse transcription-quantitative polymerase chain reaction (PCR) and immunohistochemistry assays indicated that the expression levels of serine/threonine kinase 11 (Stk11), p53 p21 and activated caspase-3 were elevated significantly in the TG-treated groups. Serine 15 phosphorylation of p53 was also enhanced significantly in the TG-treated groups. In addition, a chromatin immunoprecipitation-PCR assay revealed that the TGs induced p53 activation and enhanced the transcription of $\mathrm{p} 21$. In conclusion, TGs induce apoptosis and necrosis in rat ovarian tissues, as well as POF, via p53 phosphorylation and activation of the Stk11-p53-p21 signaling pathway.
\end{abstract}

Correspondence to: Professor Jin Zheng, Department of Gynecology, Longhua Hospital, Shanghai University of Traditional Chinese Medicine, 725 Wanpin Road, Shanghai 200031, P.R. China E-mail: jinzheng2013@126.com

*Contributed equally

Key words: premature ovarian failure, Tripterygium glycosides, p53 phosphorylation, Stk11-p53-p21 signaling pathway

\section{Introduction}

Premature ovarian failure (POF) is a typical pathological disease of the reproductive system in aging females (1-5). Patients with POF are $<40$ years old and commonly have the features of amenorrhea, hypoestrogenism and high levels of gonadotrophin (1-5). These patients also exhibit symptoms of the menopause, such as hot flushes, night sweats and vaginal dryness (1-5). Furthermore, patients with POF are usually infertile due to a lack of mature, healthy follicles or as a result of the remaining follicles not responding to stimulation (1-5); therefore, POF is also known as primary ovarian insufficiency (1-5). At present, the number of patients with POF is increasing; however, the factors contributing to POF are largely unknown.

Tripterygium glycosides (TGs) are components of the Chinese herb Tripterygium wilfordii Hook. f., also known as Huangteng (6-8). TGs possess various biological activities and can be extracted from $T$. wilfordii for use in the treatment of various diseases, such as lupus, cancer, rheumatoid arthritis and nephritic syndrome (6-8). Previous studies have demonstrated that drug resistance and toxicity of TGs can coexist (6-8). Other reports have indicated that TGs induce liver injury and dysfunction, glutathione depletion and reduce antioxidant enzymes in BALB/C mice (6-8). It is therefore necessary to identify methods of reducing the toxicity of TGs during the development of novel pharmaceuticals and clinical treatments utilizing the effects of TGs.

Serine/threonine kinase 11 (Stk11), also known as LKB1, is a serine/threonine kinase of 433 amino acids that can function as a tumor suppressor (9-11). Stk11 maintains tissue homeostasis in vivo, but Stk11 somatic mutations can be found in lung and cervical cancer (9-11). In addition, heterozygous germline mutations in Stk11 induce Peutz-Jeghers syndrome (9-11). Stk11 functions in an active complex with a pseudokinase STE20-related kinase adapter protein (STRADa or STRADb) and the scaffold protein MO25 (MO25a or MO25b) (9-11). Several substrates of Stk11 have been identified, including p53, phosphatidylinositol 3,4,5-trisphosphate 3 -phosphatase and dual-specificity protein phosphatase PTEN and serine/threonine-protein kinase 11-interacting protein. Furthermore, Stk11 activates the catalytic subunit of the 
adenosine monophosphate-activated protein kinase (AMPK) as well as several other kinases with T-loop activation domains similar to AMPK (9-11). Studies have additionally shown that heterozygous Stk11-knockout mice $\left(\right.$ Stk11 $\left.{ }^{+-}\right)$exhibit earlier tumor formation, increased tumor incidence and a reduced lifespan (9-11).

The aim of the present study was to investigate the molecular mechanism underlying the nuclear function of Stk11 in association with p53 following TG treatment. Furthermore, the study aimed to determine whether Stk11 has a direct role in the activation of $\mathrm{p} 21 / \mathrm{WAF} 1$ transcription to induce POF following TG treatment in rats.

\section{Materials and methods}

Animals and TG treatment. Hebetic female Sprague Dawley (SD) rats $(n=40)$ between four and five weeks of age were obtained from the Animal Research Center, Shanghai University of Traditional Chinese Medicine (Shanghai, China). The study was approved by the Animal Ethics Committee of Shanghai University of Traditional Chinese Medicine in compliance with the Experimental Animal Regulations of the National Science and Technology Commission of China (permit no. TCMUAE2013003). All rats were kept for 14 days, three per cage, in a temperature-controlled colony room with a standard light-dark cycle and provided food and water ad libitum. All treatment steps were performed as previously described (3-5). In brief, the animals were divided into five groups: A blank control group [healthy, wild-type (WT) SD rats; $n=8$ ] that was not treated with TGs; a negative control group (healthy, WT SD rats; $\mathrm{n}=8$ ) treated with saline; and an experimental group (healthy, WT SD healthy rats; $n=24$ ) that was subdivided into three groups for treatment with different doses (low, medium and high; n=8/dose) of TGs (Huangshi Feiyun Pharmaceutical Co., Ltd., Huangshi, China) dissolved in solution. The low-, medium- and high-dose TGs were administered every day by intraperitoneal injection $(25,50$ or $75 \mathrm{mg} / \mathrm{kg}$ body weight in $100 \mu \mathrm{ml}$ saline solution, respectively). Following treatment, the experiments using the animal model were conducted within 14 days. All of the rats were sacrificed by carbon dioxide inhalation on day 15 .

RNA extraction and analysis by reverse transcription-quantitative polymerase chain reaction ( $R T-q P C R)$. All steps were performed as previously described (5). In brief, total RNA from each cell was isolated using TRIzol ${ }^{\mathrm{TM}}$ reagent (Invitrogen Life Technologies, Carlsbad, CA, USA) according to the manufacturer's instructions. Reverse transcription of the RNA samples into cDNA was conducted using the ReverTra Ace- $\alpha$ First Strand cDNA Synthesis kit (Toyobo, Osaka, Japan). RT-qPCR was performed using a realplex 4 real-time PCR detection system from Eppendorf Co. Ltd. (Hamburg, Germany) with SYBR ${ }^{\circledR}$ Green Realtime PCR Master Mix (Toyobo) detection dye. RT-qPCR amplification was carried out over 40 cycles comprising denaturation at $95^{\circ} \mathrm{C}$ for $15 \mathrm{sec}$ and annealing at $58^{\circ} \mathrm{C}$ for $45 \mathrm{sec}$. Quantification of the target cDNA was performed using the relative quantification method. Gene expression relative to a control (calibrator) was calculated using a comparative threshold cycle $(\mathrm{Ct})$, and
Table I. Sequences of the reverse transcription-quantitative polymerase chain reaction primers.

\section{Gene}

product

Primer sequences $\left(5^{\prime} \rightarrow 3^{\prime}\right)$

\begin{tabular}{ll}
\hline Stk11 & F: CGGGCAACCTGCTCCTCACCACCAA \\
& R: CGGCAGGTGTCATCCACAGCGAAAGGG \\
p53 & F: ATGACTGAGGTCGTGAGACGCTGCCC \\
& R: GGAGCCAGGCCGTCACCATCAGAGC \\
p21 & F: AGCAGTTGAGCCGCGATTGCGATGCG \\
& R: GAGCGCATCGCAATCGCGGCTCAAC \\
18S rRNA & F: TGCGGAAGGATCATTAACGGA \\
& R: AGTAGGAGAGGAGCGAGCGACC
\end{tabular}

F, forward; R, reverse; Stk11, serine/threonine kinase 11.

steady-state mRNA levels are expressed as an n-fold difference compared with the calibrator. For each sample, the target gene $\mathrm{Ct}$ values were normalized using the following formula: $\Delta \mathrm{Ct}=\mathrm{Ct}_{\text {genes }}-\mathrm{Ct}_{18 \mathrm{~S} \text { rRNA }}$. To calculate the relative expression levels, the following formula was used: $\Delta \Delta \mathrm{Ct}=\Delta \mathrm{Ct}_{\text {treated }}$ group $-\Delta C t_{\text {control group. }}$ The values used to plot the relative marker expression were determined using the expression $2^{-\Delta \Delta \mathrm{Ct}}$, and the mRNA levels were calibrated based on the levels of $18 \mathrm{~S}$ rRNA. Gene-specific primers, as shown in Table I, were used to amplify each cDNA.

Histopathological analysis. Coronary artery tissues were stained with hematoxylin-eosin (HE) for analysis as previously described $(5,12)$. Briefly, all fresh tissues were washed three times with phosphate-buffered saline (PBS), fixed with $4 \%$ paraformaldehyde (Sigma-Aldrich, St. Louis, MO, USA) for $30 \mathrm{~min}$, dehydrated through a graded series of ethanol, cleared in xylene and embedded in paraffin. Serial $6-\mu \mathrm{m}$ sections were then cut and stained with HE.

Immunohistochemistry. Immunohistochemical analysis was performed as previously described $(5,12)$. Briefly, all fresh tissues were washed three times with PBS, fixed with $4 \%$ paraformaldehyde (Sigma-Aldrich) for $30 \mathrm{~min}$, dehydrated through a graded series of ethanol, cleared in xylene and embedded in paraffin. Serial $6-\mu \mathrm{m}$ sections were then cut and rinsed with $3 \%$ phosphate buffer, prior to microwave antigen retrieval. The primary polyclonal antibodies (Table II) were added and incubated with the sample for $45 \mathrm{~min}$ at $37^{\circ} \mathrm{C}$. Following incubation, the secondary antibody conjugated to horseradish peroxidase (Santa Cruz Biotechnology, Inc.) was added and incubated with each sample for $45 \mathrm{~min}$ at $37^{\circ} \mathrm{C}$. The avidin-biotin complex chromogenic reagent (Santa Cruz Biotechnology, Inc) was used for the secondary antibody reaction and color detection. PBS ( $\mathrm{pH}$ 7.4) was used as a negative control in the place of primary antibody. Five randomly selected fields of view (magnification, x200) from each tissue section were observed and analyzed using Intel ${ }^{\circledR}$ Integrated Performance Primitives software (Intel Corp., Santa Clara, CA, USA). 
Table II. Primary polyclonal antibodies, their source and dilutions.

\begin{tabular}{llc}
\hline Antibodies & \multicolumn{1}{c}{ Source } & Application (dilution) \\
\hline Goat anti-rat Stk11 & Santa Cruz Biotechnology, Inc., Santa Cruz, CA, USA & ICH (1:200) \\
Rabbit anti-rat p53 Ser15-pho & Santa Cruz Biotechnology, Inc., Santa Cruz, CA, USA & ICH (1:200) \\
Rabbit anti-rat p21 & Santa Cruz Biotechnology, Inc., Santa Cruz, CA, USA & ICH (1:200) \\
Rabbit anti-rat activated caspase-3 & Cell Signaling Technology, Inc., Danvers, MA, USA & ICH (1:200) \\
Rabbit anti-rat p53 Ser15-pho & Cell Signaling Technology, Inc., Danvers, MA, USA & ChIP (1:1000) \\
\hline
\end{tabular}

Stk11, serine/threonine kinase 11; Ser15-pho, phosphorylated serine 15; ICH, immunohistochemistry; ChIP, chromatin immunoprecipitation.

Table III. Hormone assay and follicle count.

\begin{tabular}{|c|c|c|c|c|c|}
\hline \multirow[b]{2}{*}{ Parameter } & \multirow[b]{2}{*}{ No treatment } & \multirow[b]{2}{*}{ Saline-treated } & \multicolumn{3}{|c|}{ Tripterygium glycoside-treated } \\
\hline & & & Low-dose & Medium-dose & High-dose \\
\hline $\mathrm{E} 2(\mathrm{pg} / \mathrm{ml})$ & $320.00 \pm 17.23$ & $310.80 \pm 11.98$ & $278.10 \pm 16.91$ & $250.10 \pm 14.65$ & $241.30 \pm 14.76$ \\
\hline FSH (mIU/ml) & $1.02 \pm 0.05$ & $1.06 \pm 0.06$ & $1.26 \pm 0.09$ & $1.83 \pm 0.14$ & $1.92 \pm 0.15$ \\
\hline Normal follicles (n) & $10 \pm 1$ & $10 \pm 1$ & $7 \pm 1$ & $3 \pm 1$ & $1 \pm 1$ \\
\hline Atretic follicles (n) & $2 \pm 1$ & $3 \pm 1$ & $5 \pm 1$ & $9 \pm 1$ & $10 \pm 1$ \\
\hline
\end{tabular}

Results are presented as the mean \pm standard error. E2, estradiol; FSH, follicle-stimulating hormone.

Chromatin immunoprecipitation (ChIP) assays. Primary antibodies (Table II) and non-specific rabbit immunoglobulin $\mathrm{G}$ (Upstate Biotechnology, Inc., Lake Placid, NY, USA) negative control antibodies were used for the ChIP experiments as described previously (9). In brief, the cells were scraped from the bottom of the culture dish, and were fixed with $1 \%$ formaldehyde for $30 \mathrm{~min}$ at $37^{\circ} \mathrm{C}$ and then quenched with $125 \mathrm{mM}$ glycine for $10 \mathrm{~min}$ at room temperature to establish DNA-protein cross-links. The samples were sonicated on ice to create 200-1,000-bp chromatin fragments and incubated with antibodies at $4^{\circ} \mathrm{C}$ overnight. PCR amplification was performed under the following conditions: 33 cycles with denaturation at $95^{\circ} \mathrm{C}$ for $30 \mathrm{sec}$, annealing at $55^{\circ} \mathrm{C}$ for $30 \mathrm{sec}$ and extension at $72^{\circ} \mathrm{C}$ for $30 \mathrm{sec}$.

ELISA assay. The rat estradiol (E2) and follicle stimulating hormone (FSH) ELISA kit (Westang Bio, Shanghai, China) was used to determine the levels of E2 or FSH in rat plasma, according to the manufacturer's instructions. Briefly, $100 \mu \mathrm{l}$ rat E2 or FSH standardarded to 0, 50, 300, 1,000, and 5,000 pg/ml, or $0,1.56,3.12,6.25,12.5,25,50$, and $100 \mathrm{ng} / \mathrm{ml}$, were added to anti-E2 FSH antibody precoated microtest wells and incubated for $60 \mathrm{~min}$. Following three washes, with ELISA washing solution, the horseradish peroxidase-conjugated detection antibodies were added, followed by the substrate solution. The absorbance was determined at a wavelength of $450 \mathrm{~nm}$ using a microplate reader (BioTek Synergy Mx; Biotek Instruments, Inc., Winooski, VT, USA).

Statistical analysis. Each experiment was performed at least three times. Data are presented as the mean \pm standard error and were analyzed using the Student's t-test when appropriate. Differences were considered significant at $\mathrm{P}<0.05$.

\section{Results}

TGs induce POF in rats. Pathology results from the treated rats indicated that TGs affect ovary function. The untreated rat ovaries exhibited an intact structure, and the ovarian interstitial cells were appropriately loose for ovulation (Fig 1). In the TG-treated rats, however, the number of atretic follicles was increased, and the ovarian granuloma cells were observed to be undergoing significant apoptosis. Furthermore, increasing the dose of TGs caused the rat ovarian granuloma cells to become more closely distributed and the number of mature follicular cells to decrease significantly. The number of apoptotic cells was observed to have increased significantly, and there was significant dysplasia in the corpus luteum. In addition, the interstitial space was generally visible at multiple bleeding sites, and inflammatory cell infiltration into the ovaries was observed in the TG-treated groups (Fig. 1). These results suggest that TGs induce damage and POF in rats.

TGs affect rat follicle maturation and hormone secretion. To characterize follicle maturation and hormone secretion, the plasma estradiol (E2) and follicle-stimulating hormone (FSH) levels, as well as follicle pathologies, were examined in mice. ELISA showed that the plasma E2 levels were decreased but the FSH levels were increased in the medium-dose TG-treated-group compared with those in the no treatment (blank control) and saline-treated (negative control) groups (Fig. 2). Furthermore, the number of follicles (atretic or 


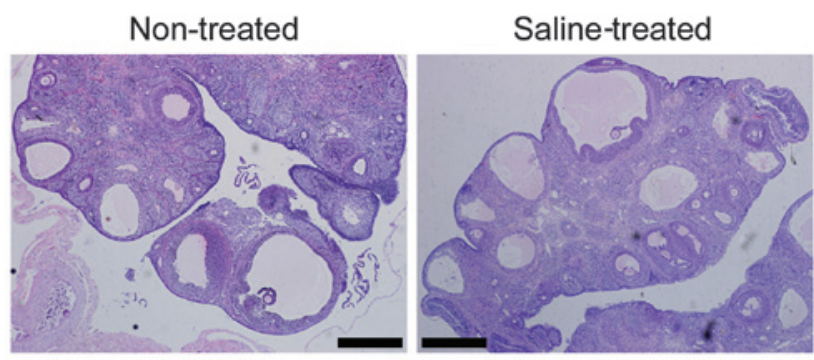

TG-treated

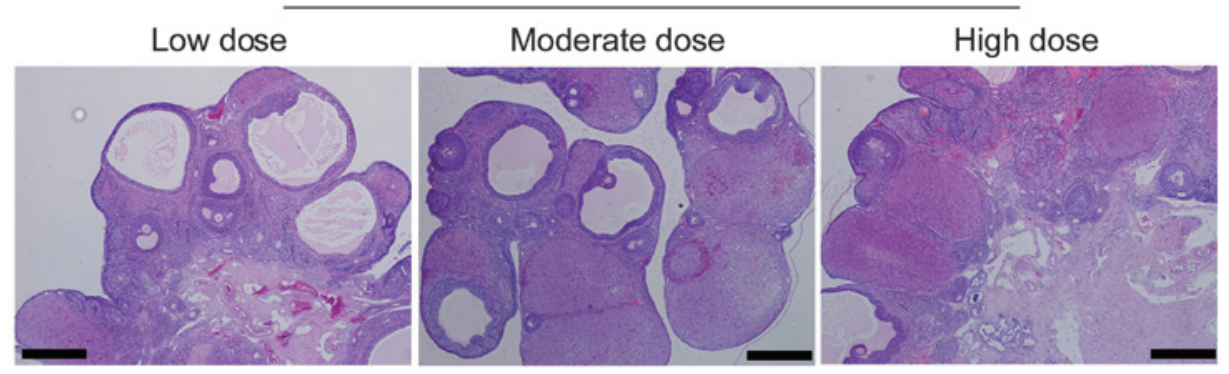

Figure 1. Pathology of rat ovarian tissues. Rats were treated with different concentrations of TGs or saline or received no treatment (non-treated), and the ovarian tissues were analyzed following hematoxylin and eosin staining. Premature ovarian failure rats had interstitial cells in a fibrous matrix, a reduced number of follicles at each stage and an increased number of collapsed oocytes following TG treatment. Original magnification, x200; scale bar, $200 \mu \mathrm{m}$. TG, Tripterygium glycoside.

A
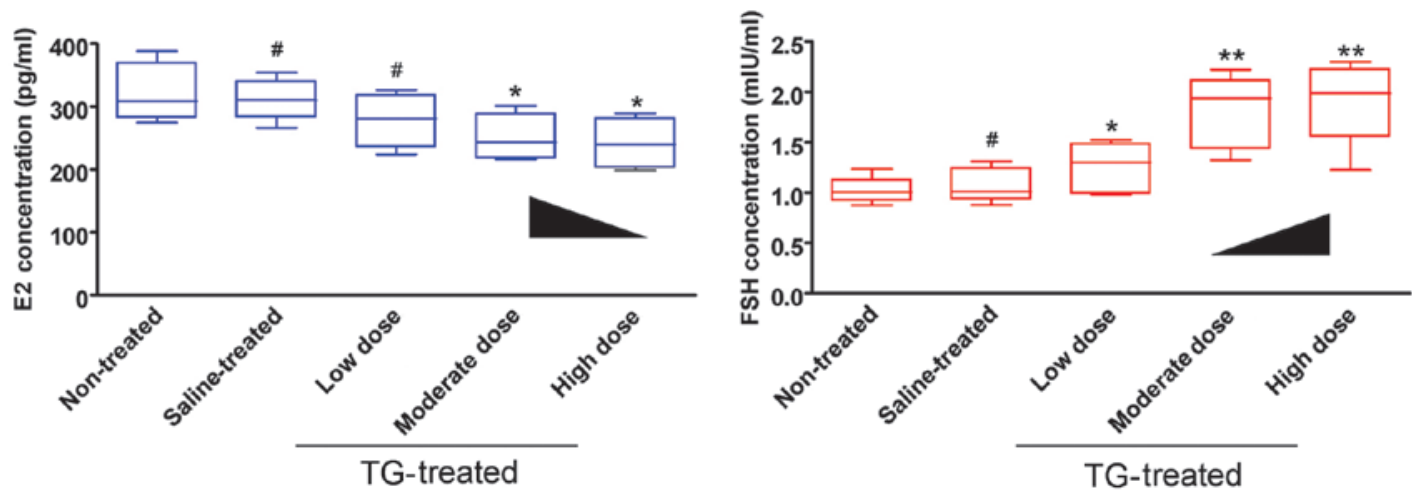

B
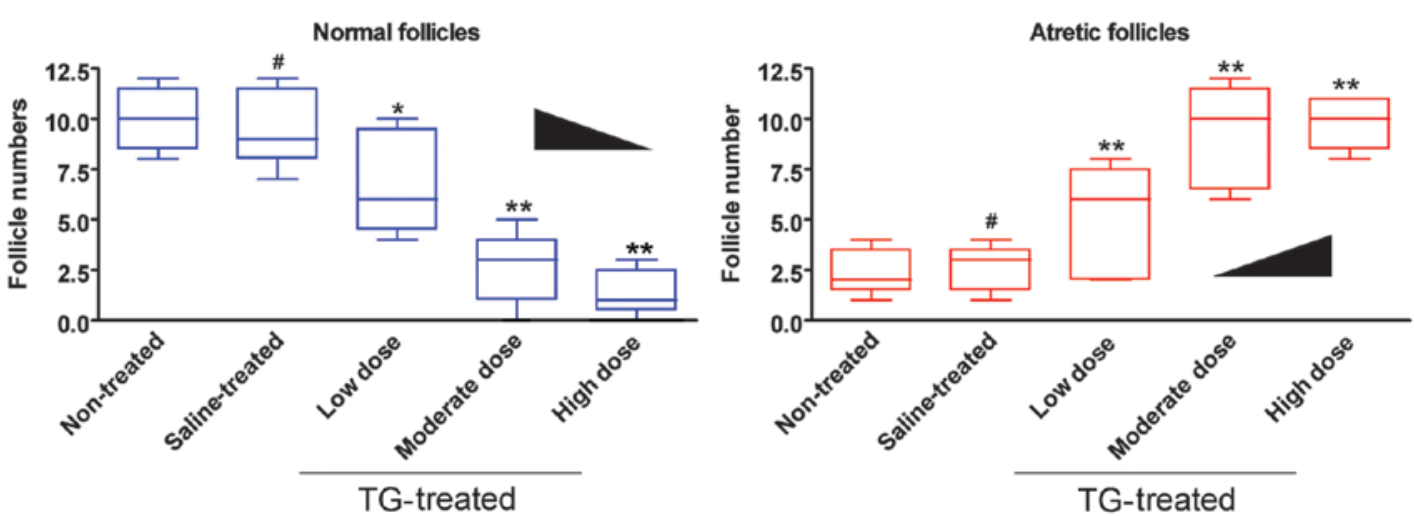

Figure 2. Plasma E2 and FSH levels and follicle count. (A) Plasma E2 and FSH levels were determined by ELISA in each group following treatment with different concentrations of TGs, saline or no treatment. ${ }^{* *} \mathrm{P}<0.01,{ }^{*} \mathrm{P}<0.05$ and ${ }^{\#} \mathrm{P}>0.05$ vs. non-treated; $\mathrm{n}=6$. (B) Follicle count revealed that there were significantly more atretic follicles in the ovaries of the POF rats following treatment with different concentrations of TGs compared with the non-treated rats. There were also fewer normal follicles in the ovaries of the POF rats following treatment with different concentrations of TGs compared with the non-treated rats. ${ }^{* *} \mathrm{P}<0.01,{ }^{*} \mathrm{P}<0.05$ and ${ }^{\#} \mathrm{P}>0.05$ vs. non-treated; $\mathrm{n}=6$. TG, Tripterygium glycoside; E2, estradiol; FSH, follicle-stimulating hormone.

normal) at every stage was counted in each group. The results revealed a significantly lower number of normal follicles but a higher number of atretic follicles in the TG-treated group compared with the non-treated and saline groups (Fig. 2 and 

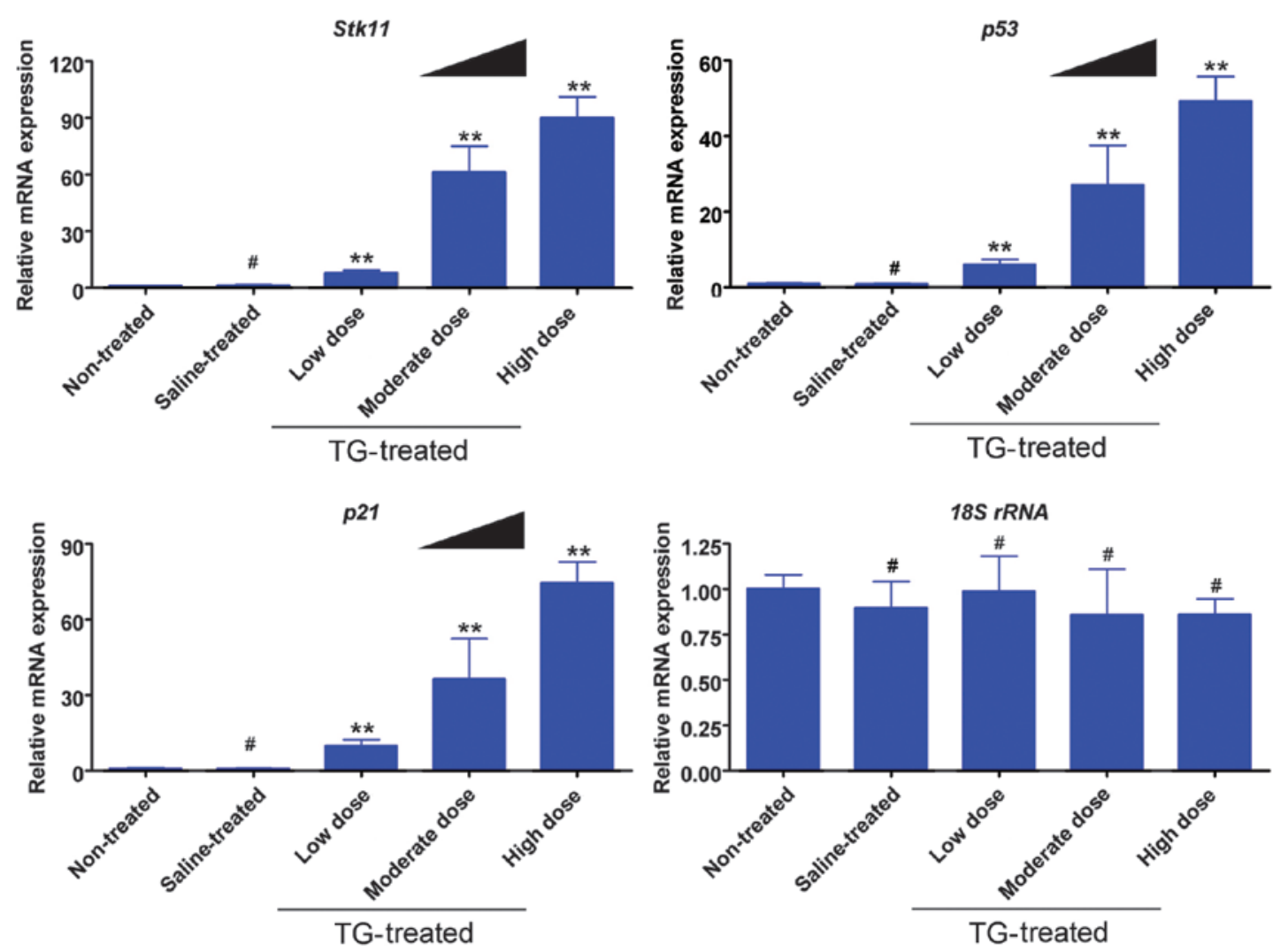

Figure 3. mRNA expression analysis of the Stk11-p53-p21 signaling pathway by reverse transcription-quantitative polymerase chain reaction. The mRNA expression levels of Stk11, p53 and p21 in the ovaries of premature ovarian failure rats following treatment with different concentrations of TGs were elevated compared with levels in the ovaries from the non-treated group. ${ }^{* *} \mathrm{P}<0.01$ and ${ }^{~} \mathrm{P}>0.05$ vs. non-treated; $\mathrm{n}=6$. TG, Tripterygium glycoside; Stk11, serine/threonine kinase 11 .

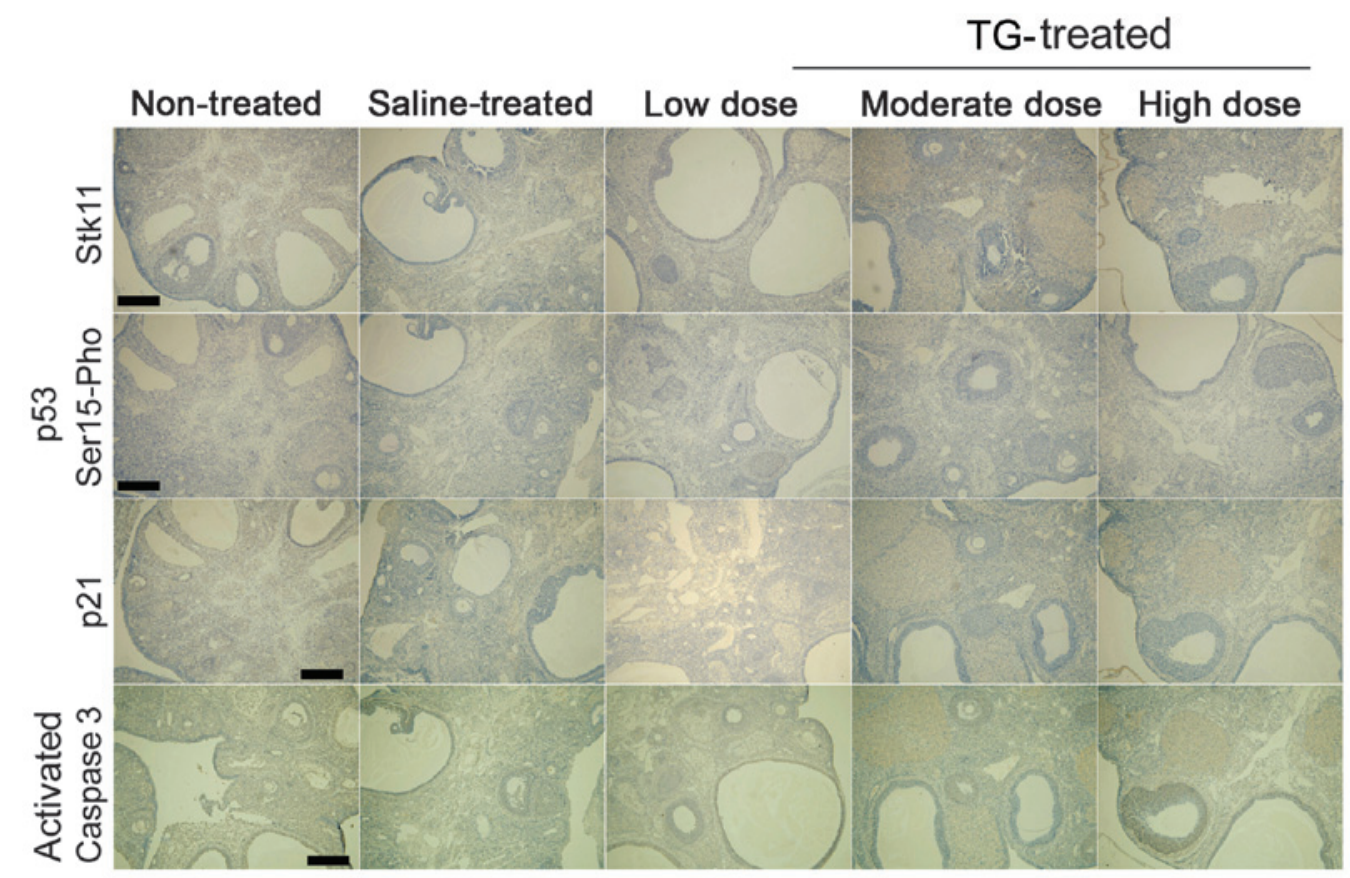

Figure 4. Protein expression of the Stk11-p53-p21 signaling pathway by immunohistochemistry. Staining indicated that Stk11, phosphorylated p53 serine 15 (p53 Ser15-Pho), p21 and activated caspase-3 were strongly expressed in the ovaries of premature ovarian failure rats following treatment with different concentrations of TGs compared with non-treated rats. Original magnification, x200; scale bar, $200 \mu \mathrm{mm}$. TG, Tripterygium glycoside; Stk11, serine/threonine kinase 11.

Table III). These results suggest that TGs induce ovarian hormonal dysregulation and follicle maturation.
TGs induce mRNA expression of the Stk11-p53-p21 signaling pathway in ovaries. The results from the RT-qPCR analysis 


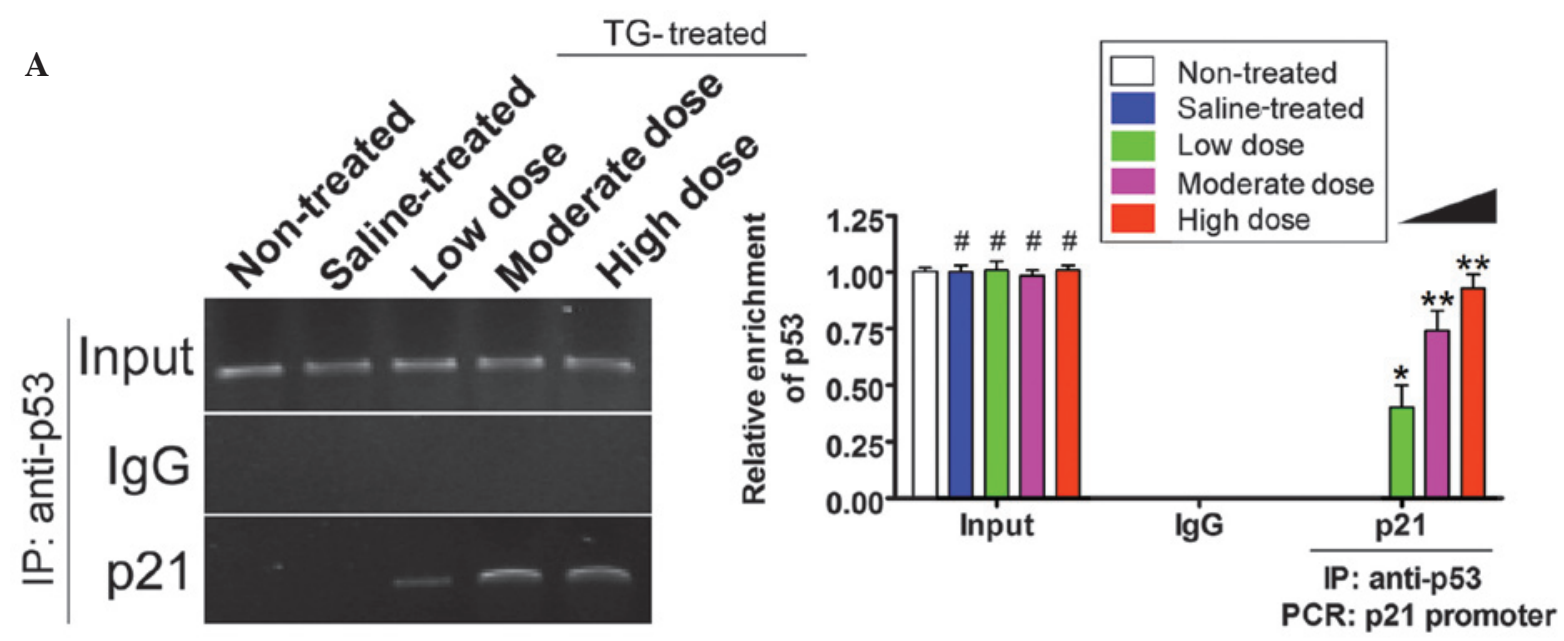

B

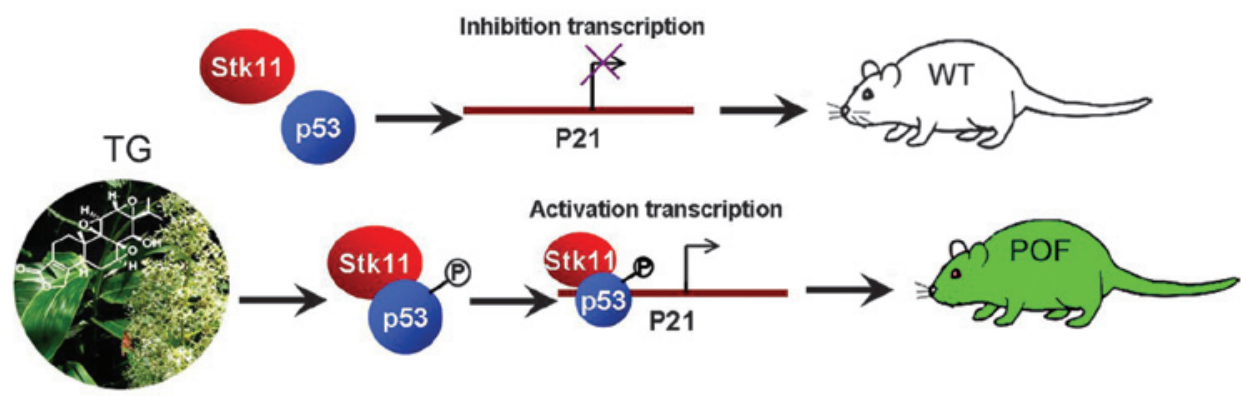

Figure 5. ChIP analysis of p53 occupancy on the p21 gene promoter. (A) ChIP assay showed significant gene amplification of the p21 gene promoter in the TG-treated groups. ${ }^{* *} \mathrm{P}<0.01,{ }^{*} \mathrm{P}<0.05$ and ${ }^{*} \mathrm{P}>0.05$ vs. non-treated; $\mathrm{n}=6$. (B) The mechanism of TG-induced POF through 53 phosphorylation and activation of the Stk11-p53-p21 signaling pathway. TG, Tripterygium glycoside; Stk11, serine/threonine kinase 11; ChIP, chromatin immunoprecipitation; POF, premature ovarian failure; WT, wild-type; $\mathrm{IgG}$, immunoglobulin G.

indicated that the mRNA expression levels of Stk11, p53 and p21 were elevated significantly in the TG-treated groups compared with those in the non-treated and saline groups (Fig. 3). Furthermore, the TG concentration was positively correlated with gene expression.

TGs induce protein expression of the Stk11-p53-p21 signaling pathway in ovaries. Immunohistochemistry showed that the protein expression levels of Stk11, p53 and p21 were significantly higher in the TG-treated mice compared with those in the non-treated and saline groups (Fig. 4). Furthermore, Serine 15 phosphorylation of p53 was also significantly enhanced in the TG-treated groups. In addition, increased TG concentration was correlated with increased caspase- 3 activation in the TG-treated groups (Fig. 4). These results indicate that TGs stimulate the Stk11-p53-p21 signaling pathway and induce apoptosis in ovaries.

TGs induce the transcriptional activity of p53. ChIP-PCR assays were used to determine the activity of the transcription factors $\mathrm{p} 53$ and $\mathrm{p} 21$. The $\mathrm{p} 21$ promoter fragments were not amplified by PCR when p53 was immunoprecipitated from the non-treated and saline groups (Fig. 5); however, in the TG-treated groups, the $\mathrm{p} 21$ promoter fragment was amplified following p53 immunoprecipitation (Fig. 5). Furthermore, this PCR product was increased with the increasing concentrations of TGs. These results therefore showed that TGs induce the transcriptional activity of $\mathrm{p} 53$.

\section{Discussion}

POF disrupts ovarian function, which causes infertility in females, but the mechanism regulating this is not clear (3-5). The cause of POF can be roughly divided into two categories: Congenital factors, such as genetic mutations, and environmental factors, including infection, inflammation, chemical drugs or radiation injury (3-5). TG is a common anti-allergy drug, which can be used for the treatment of rheumatoid arthritis, primary glomerular nephropathy, nephritic syndrome, lupus nephritis, purpura, lupus erythematosus, subacute and chronic severe hepatitis, and chronic active hepatitis. TG can also be used for the treatment of allergic cutaneous vasculitis, dermatitis, eczema, psoriasis, arthritis, leprosy, Behçet's syndrome, recurrent aphthae and ankylosing spondylitis (6-8). Although TG has certain curative effects and cost advantages in the treatment of these diseases, the side effects are becoming increasingly prevalent (6-8).

In the present study, the effect of different TG concentrations was assessed in female rats. It was found that, regardless of dose, TGs induced ovarian dysfunction and failure. The secretion of estrogen decreased in the TG-treated rats, as well as the feedback to pituitary secretion, such as gonadotropin, caused by increased FSH and luteinizing hormone. Continued treatment of TG caused ovarian tissue damage, granuloma cell swelling and necrosis, inflammatory cell infiltration and bleeding. The apoptosis of ovarian granuloma cells in the TG-treated rats was particularly evident. Based on these find- 
ings, the mechanism underlying the TG-induced granuloma cell apoptosis was investigated by characterizing the activity of the Stk11-p53-p21 signaling pathway.

Cell apoptosis or cell cycle arrest is activated when the Stk11-p53-p21 signaling pathway is expressed (9). In the present study, it was found that TG treatment in rats caused significant ovarian granuloma cell apoptosis or necrosis. Furthermore, the expression of the Stk11-p53-p21 signaling pathway in the TG-treated rat ovaries was detected. TGs not only stimulated the expression of the Stk11-p53-p21 signaling pathway in the rat ovarian tissue, particularly ovarian granuloma cells, but also induced the transcriptional activity of p53, which bound the promoter of p21 (9). When p53 binds this promoter, transcription of the p21 gene is notably enhanced (9). Activated p53 could therefore play a role in the transcription and expression of p21 following TG treatment. This may explain the toxicity of TGs and how they induce apoptosis and necrosis. In conclusion, TGs induce apoptosis and necrosis within the ovary to initiate POF via p53 activation and Stk11-p53-p21 signaling.

\section{Acknowledgements}

This study was supported by grants from the National Natural Science Foundation of China (grant nos. 81273794 and 81202811), and project funded by the China Postdoctoral Science Foundation (grant no. 2014M550250), and the Shanghai Municipal Health Bureau Fund (grant no. 20124320).

\section{References}

1. Beck-Peccoz P and Persani L: Premature ovarian failure. Orphanet J Rare Dis 1: 9, 2006.

2. Vujović S, Kanazir S, Ivović M, et al: Collagen type I alpha 1 gene polymorphism in premature ovarian failure. Srp Arh Celok Lek 141: 344-348, 2013.

3. Liu T, Huang Y, Guo L, Cheng W and Zou G: CD44 $/ \mathrm{CD} 105^{+}$human amniotic fluid mesenchymal stem cells survive and proliferate in the ovary long-term in a mouse model of chemotherapy-induced premature ovarian failure. Int J Med Sci 9: 592-602, 2012.

4. Liu T, Qin W, Huang Y, Zhao Y and Wang J: Induction of estrogen-sensitive epithelial cells derived from human-induced pluripotent stem cells to repair ovarian function in a chemotherapy-induced mouse model of premature ovarian failure. DNA Cell Biol 32: 685-698, 2013.

5. Liu T, Huang Y, Zhang J, et al: Transplantation of human menstrual blood stem cells to treat premature ovarian failure in mouse model. Stem Cells Dev 23: 1548-1557, 2014.

6. Li J, Shen F, Guan C, et al: Activation of Nrf2 protects against triptolide-induced hepatotoxicity. PLoS One 9: e100685, 2014.

7. Li XJ, Jiang ZZ and Zhang LY: Triptolide: progress on research in pharmacodynamics and toxicology. J Ethnopharmacol 155: 67-79, 2014.

8. Zhou J, Xi C, Wang W, et al: Triptolide-induced oxidative stress involved with Nrf2 contribute to cardiomyocyte apoptosis through mitochondrial dependent pathways. Toxicol Lett 230: 454-466, 2014.

9. Zeng PY and Berger SL: LKB1 is recruited to the p21/WAF1 promoter by $\mathrm{p} 53$ to mediate transcriptional activation. Cancer Res 66: 10701-10708, 2006.

10. Vaahtomeri K and Mäkelä TP: Molecular mechanisms of tumor suppression by LKB1. FEBS Lett 585: 944-951, 2011.

11. Ollila S and Mäkelä TP: The tumor suppressor kinase LKB1: lessons from mouse models. J Mol Cell Biol 3: 330-340, 2011

12. Shen DZ, Xin SL, Chen C and Liu T: Effect of atorvastatin on expression of TLR4 and NF- $\mathrm{BB}$ p65 in atherosclerotic rabbits. Asian Pac J Trop Med 6: 493-496, 2013. 